\title{
La realidad, el arte y la crítica: la poética de Ramón Gaya
}

\author{
Juan Pascual Gay \\ El Colegio de San Luis
}

\section{Resumen}

Ramón Gaya ha sido un artista que, entre sus preocupaciones, se ha interesado de manera muy visible por la realidad. Para Gaya la realidad no pude reducirse de ninguna manera al realismo o a otras corrientes y tendencias que han rebajado la realidad a una mera tesis mimética. Gaya ha criticado a quienes consideran que la modernidad ha ofrecido otras alternativas, como el arte por el arte o el arte artístico, que son capaces de desplazar el interés artístico de la realidad misma. Este texto quiere dar cuenta de lo que Gaya llama realidad y, al mismo tiempo, aclarar aquellos aspectos que considera rechazables de las nuevas propuestas artísticas.

Palabras clave: arte, realidad, realismo, arte artístico, arte creador.

\section{Abstract}

Ramón Gaya was an artist who, among other concerns, was highly interested on reality. To Gaya, reality could not be reduced in any way to realism or other flows and trends that have diminished reality to a mere Mimetic thesis. Gaya has criticized those who believe that Modernity has offered alternatives, like the art by art or the artistic art, which are able to displace the artistic interest of reality itself. This text aims to give an account of what Ramon Gaya called reality and, at the same time, to clarify those aspects of the new artistic propose that Gaya considered rejectables.

Keywords: art, reality, realism, artistic art, creation art. 
$\mathrm{C}_{\text {creciente desprestigio hacia la realidad, seguramente en una iner- }}^{\text {ste principio de siglo XXI, entre otras cosas, ha prolongan }}$ cia que ha acabado por usurpar la realidad misma en beneficio de otras "realidades" alternativas. Las consecuencias de este descrédito, así como la posibilidad de acceder a otros ámbitos que han suplantado finalmente lo que de manera tradicional se ha llamado la realidad, ha influido de modo igualmente negativo en el ejercicio de la crítica literaria, artística y cultural. Da la impresión de que la progresiva desorientación de los estudios de arte en general contribuye decisivamente a ese descrédito y a ese desprestigio. Las causas de este proceso son muchas y variadas, y el propósito de estas líneas no es tampoco el tratar de aclararlas con el fin de mostrar un listado más o menos exhaustivo de esas mismas causas para poder responsabilizarlas de una situación que de cualquier modo es la que es y que tiene pocos visos de alterarse a corto o mediano plazo; tampoco estoy diciendo que me parezca bien o mal esa situación, ni siquiera que mi opinión importe hasta el punto de alinearme a favor o en contra de una de las alternativas. De momento, me basta con señalar esta situación porque es la que me permite hablar de lo que verdaderamente me importa: la realidad en el arte y la crítica a la luz de la poética de Ramón Gaya.

Parece que si el arte está buscando nuevas vías para expresar otras realidades, la crítica no acaba de enterarse. Pero que no se entere, no quiere decir que no perciba que algo está cambiando o que, de hecho, ha cambiado ya. Desde siempre ha habido un desajuste entre la crítica y el objeto al que debe de tender, al que debe de explicar, del que debería dar cuenta. Que lo haya conseguido es otra historia, porque la crítica siempre ha vivido en una especie de desajuste, más que una inadecuación, respecto de su objeto; un desajuste que ha encontrado habitualmente dos causas precisas: o bien, no tenía bien delimitado ese objeto, con lo cual solía fracasar en su esfuerzo exegético al mostrar su incapacidad a la hora de adecuar su discurso; o 
bien, una vez que había delimitado su asunto de estudio con precisión, carecía de instrumentos solventes y rentables que dieran cuenta de ese objeto. En ambos casos, la crítica apenas se acercaba a su fin quedando de esta manera ese objeto indemne a sus esfuerzos. (Claro que estoy dando por supuestas muchas cosas: qué es la crítica, qué es el arte, qué es la realidad, pero no puedo proceder de otro modo si lo que pretendo es dar cuenta de aquello que, para mí, representa el lado más vulnerable de la crítica).

Los formalismos del siglo XX y, en particular, el estructuralismo, vinieron a poner sobre la mesa de la discusión la necesidad de una metodología parapetada en un formalismo que si bien no justificaba plenamente su pertinencia para ese objeto que pretendía iluminar, por lo menos justificaba, a sus ojos, la existencia de aquélla. Bajo los supuestos del rigor y la ausencia de ambigüedad la crítica comenzó a utilizar un lenguaje que, a la vez que construía su propia realidad formal, se alejaba del objeto que pretendía estudiar porque éste acabó por constituirse en un pretexto que permitiera el uso de aquella metodología. Así, la crítica se volvió hacia sí misma sin otro interés y sin otro alcance que el de exhibir su propia autosuficiencia sin percatarse de que, a la par, exponía igualmente sus carencias y limitaciones. Esta usurpación y manipulación de la crítica se ve de manera clara, por ejemplo, cuando defendía que el arte en cualquiera de sus manifestaciones (poesía, pintura, música, etc.) se basta y se sostiene solo; porque si el arte en exclusiva se refiere a sí mismo, su único sentido entonces reside en la teoría que lo fundamenta, con lo que, además, se justifica a sí misma. Pero si la crítica se ha disuelto en un haz de aproximaciones y posibilidades, la realidad misma en los albores de este siglo ha entrado en crisis hasta multiplicar sus sentidos y modificar sus significados. Eduardo Subirats describe esta situación: 
La intuición superrealista de Dalí fue certera: el mundo global diseñado por el nuevo artista mediático, el creador del estilo de una época o el agente organizador de la simulación electrónica de una conciencia planetaria pueden convertirse fácilmente en principio de acción y poder, de realidad objetiva y efectiva, y falsedad globalmente consensuada como piedras preciosas. Mientras tanto, lo que llamamos realidad desde el punto de vista de la experiencia individual y sus mediaciones simbólicas reflexivas, adquiere globalmente hablando el significado de lo meramente particular; una singularidad negativa y casual, y es obligada a adaptarse por medio de la violencia al sistema de simulacros mediáticos y a los poderes políticos, económicos y militares que los ampara (Subirats, 2007: 37).

La crítica se ha plegado a las exigencias de esta o estas nuevas realidades y con rapidez ha abandonado el consenso en torno a lo que entendía por realidad, que siempre remitía a la experiencia individual. Por necesidad, dar cuenta de las urgencias y exigencias de esta "falsa realidad" ha transformado al lenguaje y al discurso de la crítica en la medida que su objeto se ha transformado, hecho que si subraya la versatilidad de ésta, también revela su volubilidad, es decir, la falta de certezas sólidas sobre las cuales construir un discurso propio. Parece que la crítica, con más motivos en la actualidad, con frecuencia ha tratado de crearse un espacio para la subsistencia, más que cumplir con aquello a lo que está, en principio, destinada; también en los periodos en que había cierto consenso para aceptar la realidad o que no había otra realidad que la que se presentaba a los ojos del artista y del crítico, aunque para este último no haya en el cumplimiento de su cometido otra realidad que la que el artista convierte en arte.

Así, se produjo una paradoja que el pintor español Ramón Gaya (1910-2005) no sólo ha combatido con reiteración, sino que ha denunciado en más de una ocasión; en particular, en su ensayo ejemplar Naturalidad del arte (y artificialidad de la critica), publicado por primera vez en 1996, aunque el autor comenzó su redacción en 
Roma en $1975 .{ }^{1}$ Este breve opúsculo culminaba las reflexiones de Gaya en torno a la literatura y al arte que había realizado y publicado en diferentes medios y revistas de distintos países a lo largo de su vida; unas páginas ejemplares que encierran lo que en otros términos puede denominarse la poética de Ramón Gaya y que no ha pasado desapercibida para algunos artistas, aunque sí para la mayoría de los críticos. Los ensayos de Gaya están recogidos por la editorial PreTextos en cuatro volúmenes, en ellos puede rastrearse el orden cronológico y los intereses que le llevaron a la escritura de Naturalidad del arte. Merece destacarse un texto intitulado "Fragmento de un escrito inédito", (1992: 17-22) fechado en Espańa en 1983, donde el autor apunta algunas ideas y reflexiones que más tarde incorpora, dotándolas de envergadura y personalidad, al ensayo citado. Este "Fragmento" comienza de la manera que sigue:

Puede, eso sí, juzgarse lo que hacemos, pero no lo que somos —y aquí es donde se encuentra el nudo de la cuestión-, pues la verdad es que la poesía, la música, la pintura, la escultura, no son en absoluto, como se ha dado por descontado siempre, actividades, las muy bellas y elevadas actividades de ciertos seres de excepción - los artistas-, sino iniciativa, pasiva naturaleza carnal, animal del hombre, del hombre... común.

No haber visto, no haber comprendido el carácter "común" del arte creador, del acto creador, es lo que más contribuye a desviarnos de su naturaleza verdadera, de su verdadera identidad, de su razón de ser, ya de por sí escondidas y misteriosas. El arte ha sido visto siempre como la meritoria inclinación de unos cuantos - de esa clase especial de hombres que llamamos artistas— (Gaya, 1992: 19).

Estas líneas recogen las ideas que cohesionan y en torno a las cuales se construye Naturalidad del arte: el arte no es una tarea destinada a

${ }^{1}$ Para este artículo utilizo la edición de Pre-Textos de 2001 (ver bibliografía). 
seres excepcionales, sino que conviene a la gente común; el arte es una "actividad" plenamente humana, una extremosidad del hombre, cuya causa reside en la naturaleza humana y no en otra cosa que esté fuera de esa condición; finalmente, el haber entendido el arte como un acto excepcional ha contribuido de manera decisiva a no comprender en absoluto qué es el arte. Sin duda, estas certidumbres de Gaya han afectado, en su opinión, la percepción del arte por parte no sólo de los artistas sino del público. Pero esta desviación respecto a la manera de entender el arte ha tenido otras consecuencias también negativas. Así, la primera consecuencia de esta falta de atención a la verdadera naturaleza del arte ha afectado a la crítica artística que ha desvirtuado no sólo el arte sino la imagen de los artistas. Gaya, sin decirlo de forma explícita, arremetía contra la idea del genio artístico de origen romántico y al socavar el concepto de genialidad traía, en realidad, al primer plano de la discusión la vieja dicotomía entre el artesano y el artista; una dicotomía que se polariza durante las vanguardias al relegar la técnica y la destreza artísticas, en beneficio de la acción individual de éste sobre los materiales con los que hace su obra o, dicho de otra manera, cuando lo importante no es el cómo sino el quién. Arrumbar la técnica era una manera de abolir la tradición en la que ese arte se insertaba y, por tanto, un medio no sólo para borrar la memoria sino para hacer tabula rasa del pasado como ventilaba y exigía el Primer manifiesto surrealista. El artista del que habla Gaya está a medio camino entre el artista moderno y el artesano. Se puede establecer una analogía elocuente entre el artista y el artesano: si el ojo le corresponde al artista, es la mano la que es propia del artesano. Al hablar del artesano, Gaya dice que es importante la mano, que no basta con una mirada o una idea original o genial, sino que es necesaria una ejecución técnica suficiente. Escribe Richard Sennett:

El artesano explora estas dimensiones de habilidad, compromiso y juicio de una manera particular. Se centra en la estrecha conexión 
entre la mano y la cabeza. Todo buen artesano mantiene un diálogo entre unas prácticas concretas y el pensamiento; este diálogo evoluciona hasta convertirse en hábitos, los que establecen a su vez un ritmo entre la solución y el descubrimiento de problemas (Sennett, 2009: 21).

Y la mano, además de expresar el oficio, representa la memoria de ese oficio. Sin pasado y sin memoria el arte se volvió hacia sí mismo para ofrecer lo que Ramón Gaya y, más tarde, Tomás Segovia denominan un arte artístico. Escribe el pintor-poeta:

(La decisión que se tomara, al empezar el siglo XX, de procurarnos a toda costa un arte [...] en sí mismo, desasido, desentendido de la realidad —un arte inventado, ideado, imaginado, fantaseado, colocado encima, pegado encima, puesto, superpuesto, postizo, añadido, o sea, un arte, cuando mucho, pergeñador, confeccionador de cosas-, pudo parecer entonces, hace setenta y tantos años, una vívida acción purificadora, salvadora, que nos libraba para siempre del tontísimo y tristísimo realismo, pero nos damos cuenta hoy, a la vista de tanta basura artificial como ha ido acumulándose, que era tan sólo una decisión estúpida, y también, quizá, un tanto... satánica, juguetonamente satánica, de un satanismo estéril, infantil, pueril (Gaya, 2001: 14-15).

La crítica a la realidad es la premisa sobre la que se erige el nuevo arte, pero esta crítica, según Gaya, padece de miopía al rebajar la realidad a lo que, en arte también, se ha llamado realismo mediante un reduccionismo que en lugar de afectar a la poética de ese movimiento acabó por desprestigiar a la realidad misma; un baldón referido a la escuela antes que a la materia en que supuestamente se sustentaba que terminó por desacreditarla, porque el realismo pecaba de ingenuidad y simplismo, mientras que la realidad se asienta en la presencia y la verdad; verdad y presencia que nunca son ni ingenuas ni simplistas 
puesto que se agotan a sí mismas sin otros calificativos. Importa volver sobre el realismo como una poética, una manera de hacer y entender el arte, que rebaja según Gaya la realidad. Para Gaya, como se ha dicho, realidad no es sinónimo de realismo. Señala Juan Oleza que en 1847, en España, se inicia una segunda fase del debate en torno al realismo "a la francesa":

En esta segunda fase del debate el núcleo central lo constituye la defensa o el rechazo de las diversas alternativas propuestas al realismo "a la francesa": el arte por el arte, el realismo idealista o el idealismo realista, el arte docente. Esta segunda fase se proyectó sobre el debate en torno al naturalismo, como muestran los Apuntes sobre el nuevo arte de escribir novelas (1886-1887), de Juan Valera, quien prolonga contra el naturalismo de los ochenta sus ataques de los años sesenta y setenta contra el realismo de los Feydeau, Flaubert y Champfleury (Oleza, en Romero, 1998: 420).

El asunto de fondo para el realismo era que si la literatura y el arte expresan no un tiempo eterno, sino un tiempo histórico, entonces el realismo sería la expresión misma de la modernidad. Los discursos de la época, como los de Montoro o Moreno Nieto, incidían en que el realismo era la alternativa más fiable para dar cuenta del arte contemporáneo, dada su naturaleza y los nuevos modos de vida en el último cuarto del siglo XIX (Oleza, en Romero, 1998: 421). En realidad, el discurso de Gaya está dirigido a las dos posiciones que polarizan a finales del siglo XIX el debate en torno al arte: la primera es la que corresponde a la tradición idealista y romántica, presidida por la estética hegeliana; la segunda es la respuesta a esa estética. Si la primera privilegia el arte por el arte, el arte artístico en términos de Gaya, la segunda arremete precisamente contra este arte por el arte, aun cuando sus detractores pertenecían a posiciones ideológicas enfrentadas (Ver López-Morillas, 1956; López, 1977; y Miralles, 1979). Ramón Gaya no se refiere al hablar de realidad ni al realismo ni al arte por 
el arte: respecto del primero, porque supone una degradación de la realidad tal y como él la entiende; en relación con el segundo, porque nada hay más alejado del arte que el arte vuelto sobre sí mismo.

Pero lo que los artistas no advirtieron, tampoco la crítica, es que la realidad no puede degradarse por ningún medio porque el arte, por muy realista que sea, ni la toca ni la roza, ya que siempre permanece indemne a la acción del artista a partir de ella. Para Gaya, la realidad hay que entenderla en la tradición de la sacralización de la realidad según Rudolf Otto, quien escribe:

Eran considerados como "señales" todos los aspectos y circunstancias de que hemos hablado anteriormente: lo terrible, sublime, prepotente; lo que sorprende y, de manera muy especial, lo enigmático o incomprendido, que se convierte en portento y milagro. Pero hemos visto que todas estas circunstancias no son "señales" en estricto sentido, sino coyunturas y motivos ocasionales para que el sentimiento religioso se despierte espontáneamente. Lo que en ellas produce este efecto estriba en ciertos aspectos que tienen análogos con lo santo (Otto, 2001: 183).

Esta es la idea de realidad de la que participa Gaya: las características apuntadas por Otto forman parte indivisible de la realidad; una realidad santa que poco o nada tiene que ver ni con el realismo, ni con el arte artístico, en nada parecido al arte creador defendido por el ensayista español que siempre es un trasunto de la realidad. Así, dice también Gaya:

El hacedor de arte, sin duda muy cansado y asqueado de esa imagen tan falsa que de la realidad nos venía dando el "realismo" a lo largo de todo el siglo XIX, quiso reaccionar con violencia, sin contemplaciones, y, como suele suceder cuando se está demasiado cegado por la razón, al arremeter contra el realismo, arremetería, de paso, sin darse cuenta, contra la misma realidad — la santísima realidad_-, o 
sea, confundiéndose, embarrullándose. Mala suerte; porque todo ese infeliz arte moderno que se nos avecinaba sería, pues, edificado, levantado sobre un error; en nombre de un error, un error... sacrílego, un error que no era simplemente un error, sino que llevaba dentro una profanación muy necia y muy grotesca (Gaya, 2001: 15-16).

Gaya pone en estas escasas líneas los puntos sobre las íes respecto a lo que entiende como la cualidad más definitoria de la realidad. No sólo puede cometerse sacrilegio contra la realidad sino que puede profanarse. Sacrilegio y profanación son vocablos del ámbito religioso y, más precisamente, sagrado. Para Gaya la realidad no sólo es "santísima” sino también sagrada. Y lo sagrado es lo único sobre lo que puede cometerse sacrilegio y profanarse. La realidad es, pues, sagrada y su negación, como pretende el arte artístico es un sacrilegio, una profanación. Por eso, si el arte tiende por naturaleza hacia la realidad, no hacia el realismo, el artista no puede ser ese ser excepcional o ese genio que el romanticismo se encargó de ensalzar y encumbrar, sino el hombre común y corriente, ese que es capaz de recibir la realidad "entera y verdadera”, porque el artista verdadero no copia la realidad sino que la revela: "Sólo el hombre común —el "superhombre común”-, es decir, el hombre común... sano, limpio, fuerte, silvestre, puede y sabe recibir la realidad entera y verdadera, sin manosearla ni manipularla, sin la boba idolatría de unos, ni la ilusa y petulante alteración o deformación de otros" (Gaya, 2001: 14).

El realismo copia —o lo intenta- la realidad, mientras que el verdadero artista la revela, pero revela eso que de sagrado y santo hay en ella; es la sacralización de la realidad la que hace, según Gaya, que el único deber del artista sea el desvelamiento de su carácter sagrado. La sacralidad del arte reside precisamente en su naturalidad y es este carácter el que denuncia al mismo tiempo lo artificioso de la crítica artística, porque la crítica no entiende plenamente el arte, es decir, no comprende de lo que paradójicamente trata: "Lo más patético del 
crítico de arte — de música, de poesía, de pintura — no es tanto que se equivoque y no entienda, sino que entiende de una cosa que... no comprende". La distinción es relevante: "entender es tener idea clara de las cosas o saber con perfección una cosa", mientras que comprender es "abrazar, ceñir, rodear por todas parte una cosa”; si entender es un proceso exclusivamente de la razón, comprender, además de ser un acto, lo es también del cuerpo; si el primero compete únicamente a la inteligencia, el segundo al ser entero. Por eso Gaya insiste una y otra vez en la animalidad o carnalidad que representa el acto artístico, características que el crítico, en principio, ni puede sentir, ni puede expresar, sometido como está a la razón:

Pero comprender es otra cosa. Comprender es un acto más bien seco y rotundo, muy rápido, además, pues a la sola aparición del sujeto en cuestión, éste debe ser instantáneamente comprendido por nosotros de un solo golpe, de una vez por todas, o no lo comprenderemos nunca. [...] Entender de arte — de música, de poesía, de pintura-, actuar de entendido, de perito, de crítico en fin, no puede ser más, en el mejor de los casos, que una simple ocupación rara, o sea, algo que se hace, pero que no se es; el crítico no nace, se hace; el puesto del crítico es una de esas necesidades artificiales que de tanto en tanto inventa la ajetreada sociedad, pero en donde la naturaleza viva no ha tomado parte (Gaya, 2001: 10-11).

Gaya plantea una nueva dicotomía en torno al arte y la crítica, pero en este caso definitiva: definitiva porque el arte viene del ser, mientras que la crítica del hacer; definitiva porque, por tanto, el arte es natural, a diferencia de la crítica que es artificial; definitiva porque el arte vive frente a la crítica que, más bien, amortaja. Este reconocimiento, el hecho de que el arte sea vida, sitúa la verdadera distancia con la crítica, puesto que "la vida no puede ser objeto de crítica; (quizá pueda, eso sí, someterse a la crítica el... mundo, todo aquello que es mundo, mundanal ruido, pero no la vida) la vida no puede ser 
espiada, indagada, investigada, juzgada, ni siquiera entendida, sino comprehendida, aprehendida" (Gaya, 2001: 12). La crítica, pues, es incompetente por naturaleza para enfrentar el acto artístico, pero a ojos de Gaya esta evidencia no es lo peor que le sucede a la crítica, sino que no reconozca esta incompetencia desde el principio y por tanto haya usurpado un espacio que no le corresponde en modo alguno, hasta desplazar, en ocasiones, al arte mismo. Tal vez, la crítica sea un ejercicio que se ajuste a la naturaleza del arte artístico, es decir, del arte vuelto hacia sí mismo, pero no del arte creador. Esta nueva división es importante también para Gaya:

La poesía, la música, la pintura, han sido siempre realizadas por unos pocos, sí, pero en nombre de todos. Si se hubiese tenido en cuenta que el arte creador — no el arte artístico, ya que éste sí va destinado y dado a un público - no se ha hecho jamás para unas gentes, sino en lugar de ellas, nos habríamos evitado tanta palabrería sobre arte social, o minoritario, o revolucionario, o aristocráticos, o burgués, o puro, o útil, o... moderno. El arte creador, hacedor de criaturas, no se dirige a nadie ni a lugar alguno conocido; podría decirse que la creación no va a ninguna parte, sino que... viene, viene de muy lejos y muy dentro hasta alcanzar una superficie real, de la realidad (Gaya, 2001: 16-17).

Estas líneas subrayan por lo menos dos aspectos relevantes relacionados con el arte y el artista: el arte, cuando se trata del arte creador, no está dirigido a nadie ni a nada, no busca un público y mucho menos un mercado, así el artista no puede considerarse ese ser extraordinario que nuestra sociedad se ha encargado de repetir hasta la saciedad, sino precisamente ese ser común que es capaz de desvelar artísticamente la realidad; así el artista, más que actuar sobre la realidad, es ese sujeto que es capaz de interpretarla, capaz de recibir lo que la realidad puede darle, más cerca de un transmisor, un transmisor cualificado si se quiere, que de un productor. Gaya parece negar sistemáticamente 
las aportaciones de la modernidad al arte y, más bien, busca en el periodo anterior, en particular en el Renacimiento italiano, el estado del artista y, también, el de la crítica. En un texto intitulado "Belleza, modernidad, realidad", fechado en 1960 en Italia, Gaya muestra lo que entiende por modernidad, que nada tiene que ver con lo que los demás entienden acerca de ésta, aunque sus críticas se dirijan a la idea convencional y no a lo que él entiende por tal:

Hay algo muy atrevido - como casi siempre sucede con el genio italiano- en esta arquitectura... "sabida"; algo muy nuevo, muy fresco, muy reciente, sin caer en la patética "originalidad". Es lo que nunca deja de sorprenderme en Palladio: su "modernidad" segura, tranquila. No creo que Goethe buscara en él —como supone- lo antiguo - $\mathrm{o}$ antiguo convertido en fantasma que vuelve-, sino una modernidad vigorosa, firme, que abarcara también lo antiguo, que fuese por fin "estable", y no esa caricatura movida, frívola que intentan imponernos (Gaya, 1992: 83).

Para Gaya la modernidad no es un concepto de la historia del arte, ni siquiera de la periodización, sino que es la actualización de ese continuo que reside en el arte que comprende la realidad, que abraza la realidad, que es. Modernidad o moderno en la poética de Gaya significa actual, pero una actualidad que no abole el pasado sino que lo asume y lo restablece: la modernidad, por ejemplo, de una pintura de Picasso es igual en términos de arte a la de Giorgione o Velázquez o Goya. Para Gaya, en términos históricos, no existen las divisiones, ni los periodos, ni las fragmentaciones temporales más o menos motivadas o más o menos arbitrarias: la modernidad es el arte de siempre que en su calidad de vida, como la vida misma, llega hasta el ahora; por eso el arte "viene, viene de muy lejos", hasta hacerse presente en la realidad que es el arte creador. En este sentido, de la manera como la entiende Gaya, la modernidad es vida, pero no como suele considerarse habitualmente, como un periodo histórico que nace a la par con la industrialización: 
Esa clase de modernidad tan viva — que muy a menudo nos parece reconocer en lo italiano- es más bien, me atreveré a decir, como una... salud, como una "salud indomable". Es difícil caracterizar una cosa que, sin dejar de ser ella, es otra, y aun otras, y no propiamente una suma de las mismas, pues se trata de algo indefinible y un tanto borroso, pero también muy "decisivo" para poder llegar sin reservas, nosotros españoles, hasta "el descarado" arte italiano; es difícil caracterizar una cualidad tan evidente como vigorosa, pero si no sabemos darle un nombre, no podemos, por otra parte, ignorarla, pasarnos sin ella, pues "no explica", pero sí "lo ilumina todo", permitiéndonos entonces ver, entrar, en lugar de quedarnos fuera, malhumorados y... "juzgando" sin remedio. Pronto me pareció descubrir que aquella impertinente salud era, sin duda, lo que derrama sobre el arte italiano esa rara modernidad incesante. Y no sólo Modernidad y Salud parecían aquí fundirse, sino que Clasicismo, Belleza, Vitalidad, Misterio, Alegría, venían también a fortalecer y colorear esa extraña sustancia única, afirmativa, presente, que empapa todo lo italiano como una niebla clara (Gaya, 1992: 83-84).

Gaya recupera cierto vitalismo de la filosofía de José Ortega y Gasset y se alinea decisivamente con María Zambrano, quien en los primeros párrafos del ensayo "Pensamiento y poesía" establece una distinción en términos parecidos a la que asienta Ramón Gaya entre el arte artístico y el arte creador:

A pesar de que en algunos mortales afortunados, poesía y pensamiento hayan podido darse al mismo tiempo y paralelamente, a pesar de que en otros más afortunados todavía, poesía y pensamiento hayan podido trabarse en una sola forma expresiva, la verdad es que pensamiento y poesía se enfrentan con toda gravedad a lo largo de nuestra cultura. Cada una de ellas quiere para sí eternamente el alma donde anida. Y su doble tirón puede ser la causa de algunas vocaciones malogradas y de mucha angustia sin término anegado en la esterilidad. 
Pero hay otro motivo más decisivo de que no podamos abandonar el tema y es que hoy poesía y pensamiento se nos aparecen como dos formas insuficientes; y se nos antojan dos mitades del hombre: el filósofo y el poeta. No se encuentra el hombre entero en la filosofía; no se encuentra la totalidad de lo humano en la poesía. En la poesía encontramos directamente al hombre concreto, individual. En la filosofía al hombre en su historia universal, en su querer ser. La poesía es encuentro, don, hallazgo por gracia. La filosofía busca, requerimiento guiado por un método (Zambrano, 1996: 13).

Dejando de lado las alusiones a la filosofía que en el caso de Gaya no parecen contar mucho, las referencias de Zambrano a la poesía no sólo coinciden en la carnalidad que también emplea el pintor para calificar en encuentro con el arte, sino en el tono ensayístico y en el uso de términos semejantes. Pero conviene apuntar una distinción extrema: para Gaya la razón, cuando adquiere todo el protagonismo, aleja al hombre de la verdadera naturaleza del arte; no es que sea antiracionalista, sino que la razón debe participar en la creación sin opacar los otros aspectos del hombre. Por eso, en parte, su visión negativa de la crítica artística que únicamente se sirve de la razón para tratar de dar cuenta de un objeto, el artístico, en que ésta no sobresale por encima de otras potencias, sino que se amalgama y confunde y abraza con ellas; por eso el crítico "entiende" pero no "comprende":

Alguien podría decir que existe en el hombre un cierto "sentido crítico" innato, parte integrante y congénita de su ser, pero un "sentido", como sabemos, es algo muy personal y, aunque sumamente importante, tan sólo de carácter... auxiliar. El sentido crítico no puede ser más que una herramienta personal, íntima, casi secreta, de trabajo; pero es ese "trabajo", precisamente, el que debe cesar, interrumpirse en el instante mismo que, del cuerpo de tal o cual obra, veamos elevarse el oscuro milagro de una criatura verdadera, completa, viva; porque ante la vida no podemos, necia y porfiadamente, seguir siendo críticos, actuar como críticos (Gaya, 2001: 12). 
La razón no puede dar cuenta, en opinión de Gaya, del arte, del verdadero arte, porque el verdadero arte es, vive, y la vida así entendida es reactiva al escrutinio y al juicio puesto que ésta es sagrada y lo sagrado se acepta sin más por su misma naturaleza. La realidad es santa porque es una manifestación palpable, visible, de la vida: porque es el primer ámbito en el que el sujeto se relaciona con la vida, por eso la realidad no es únicamente lo que se toca o lo que se ve o lo que se oye, es todo eso, pero también mucho más; es este mucho más lo que revela el arte creador, a diferencia del arte artístico que no desvela nada porque está encerrado en sí mismo, vuelto sobre sí mismo, plegado en sí mismo, por eso es estéril y baldío. Esta reflexión sitúa a la crítica en otro lugar al que habitualmente ocupa. Así, la crítica artística de Ramón Gaya — porque también ha ejercido la crítica— no pretende como sí hace la mayoría, explicar o enseñar, sino acompañar para ver tal o cual cuadro. Tomás Segovia advierte con clarividencia la diferencia entre explicar y acompañar, precisamente a propósito de Gaya:

Comentar por escrito los cuadros de Ramón Gaya es por eso una tentativa especialmente desplazada: sus Homenajes nos están diciendo qué clase de comentario sería el que podría enriquecer nuestra visión, no nuestro conocimiento. Y sin embargo, él mismo ha hablado también, por escrito, de muchos pintores, de muchos cuadros. Sí, pero en primer lugar, escribir sobre un cuadro o un pintor al que se enfrenta al mismo tiempo con los pinceles es escribir desde una dignidad que no puede tener el comentarista que mira ese toro desde la barrera; y en segundo lugar, los comentarios escritos de Ramón Gaya son también del orden del homenaje mucho más que de la crítica; tienen el tono y la libertad de esos comentarios inspirados que mencionaba más arriba, que son ante todo un episodio de nuestra vida más honda y sólo de rebote, cuando lo son, piezas de una explicación pretendidamente neutra (Segovia, 2000: 138). 
No le falta razón a Segovia, la crítica artística de Ramón Gaya, es un ejercicio que busca más acompañar al espectador o al lector, que enseñarle o explicarle aquello que se le presenta a los ojos; o como diría él, más que penetrar aquello que tiene ante sí el espectador, busca mostrarlo para, luego, abrazarlo. Podrían utilizarse otras palabras de Segovia, en este caso dirigidas a la poesía de Juan Ramón Jiménez, para expresar cabalmente lo que Gaya en su crítica:

Porque al hablar de una obra poética, el lenguaje del entusiasmo, el lenguaje puramente exclamativo que nada predica, ni describe, ni concluye, es el único que puede estar seguro de no usurpar el lugar de la obra, de no sustituirla por una traducción fatalmente mucho más pobre, pero que también fatalmente aparece como más viable o incluso más verdadera en el sentido de una verdad utilizable. El pasmo y el regocijo son en rigor las únicas actividades que no desplazan al poema (Segovia, 2000: 15).

Conviene sustituir en la cita anterior los vocablos poética y poema por artística y arte, de manera que esta cita podría aplicarse igualmente a lo que Gaya entiende por función de la crítica artística. Eso que dice Segovia que quiere hacer con la poesía de Juan Ramón Jiménez es exactamente lo mismo que Gaya hace con su crítica de arte, y al leer a Segovia cuando habla del pasmo y la mostración, por momentos, es como si estuviéramos leyendo a Gaya:

El pasmo es otra cosa. No puede ser terminal porque no salir del pasmo es caer en la parálisis y la muerte. Es un paso momentáneo por la muerte, una parálisis pasajera y purgativa, una suspensión de las certezas para volver a arrancar rejuvenecido. Está emparentado con la pura mostración, como sugerí al principio. Una de sus figuras es ese momento en que nos topamos por sorpresa con algo o con alguien muy deseado, y lo único que podemos hacer es abrir la boca y señalarlo con el dedo. Pero es claro que ahí no termina, sino 
que empieza, el encuentro; después viene el abrazo. Esta figura es también una figura de respeto. El pasmo que no toca lo deseado, el abrazo que no lo deja sumido en sí mismo, vienen antes y después y ni lo usurpan y ni lo clausuran (Segovia, 2000: 16).

La crítica es para Ramón Gaya del orden de la mostración. Esta capacidad del pintor para no enseñar sino para conseguir que aquello acerca de lo que habla aparezca, emerja de pronto, irrumpa, pero no porque lo trae a la página, si no porque eso que evoca se deja venir, es característica también de sus evocaciones, como por ejemplo el texto intitulado "Merced 22":

¡Hace 50 ańos! ¡Medio siglo! Murcia era entonces, todavía, Murcia, concentradamente Murcia. Cada lugar de Espańa era entonces todavía él, muy él y ningún otro, es decir, cada sitio era un sitio único, singular, y no sólo por su carácter o fisonomía diferentes, sino por su... sustancia, por su solitaria sustancia. España, la invertebrada, era, pues, entonces, como un tapiz muy rico y muy apretado de... "soledades juntas". Pero así como ser Córdoba —o ser Toledo, o ser Valencia - era ser una singularidad bastante dibujada, ser Murcia era ser una singularidad mucho más imprecisa, más misteriosa, más secreta, más fina (sí, más fina), más inefable, más indecible, más invisible (Gaya, 1992: 103).

El escritor, Ramón Gaya, describe una ciudad, Murcia en este caso, pero no en términos geográficos que sería lo previsible, sino en términos espirituales. $\mathrm{Al}$ ofrecer este retrato anímico de una ciudad, muestra su proceder no sólo en la crítica de arte, sino a la hora de comprender esta actividad. No se trata de penetrar determinado asunto para tratar de aclarar y aclararse, sino que deja que ese objeto vaya apareciendo pero a partir de aquello que físicamente es, sino de aquello que actúa como una sustancia que lo impulsa hacia el presente: Gaya no evoca, da la impresión de que el recuerdo vaya emergiendo, 
pero tampoco es el recuerdo propiamente, sino aquello que hace de Murcia lo que es y no lo que parece ser. Este parecer ser es lo que habría ocupado a una escritura realista, pero no a un escritor, como es el caso, atento a la realidad. Tal vez por eso, la evocación de Murcia, que no es propiamente una evocación, sino más bien una emergencia, está condicionada por el uso de sustantivos y no de adjetivos, cuando paradójicamente el recuerdo suele ser descriptivo. El predominio de sustantivos resulta elocuente de un proceso de revelación antes que descriptivo.

Ramón Gaya es un artista-pensador por lo menos interesante, sobre todo si se recuerda que nunca recibió una formación académica de ninguna clase. Su pensamiento y su reflexión nacen de su práctica como pintor y poeta. Pero por lo menos tuvo el acierto de que esa práctica no le dejara indiferente en términos intelectuales, sino que esa reflexión alentó y enriqueció su quehacer artístico. Tampoco puede pensarse que su pensamiento es un caso excepcional en el siglo XX hispánico, más bien sigue las huellas impresas por Ortega y Gasset y camina de la mano de María Zambrano. Tal vez por eso no sorprende su lenguaje religioso, místico en ocasiones, y tampoco su mirada absorta y perpleja hacia la realidad como expresión del ser. Esta perplejidad es la que denuncia y reconviene a la crítica al uso, incapaz de comprender otra realidad que no sea la del objeto artístico en cuanto objeto, pero no como trasunto de la verdadera realidad. Para Gaya, parece que la razón de la crítica es el acompańamiento del arte, pero sin violentarlo ni intimidarlo, por la sencilla razón de que si es arte verdadero, arte creador, realidad revelada, por mucho que pretenda o quiera esa crítica nunca dará cuenta de éste, no sólo porque se trata también de dos lenguajes, sino porque también obedecen a dos ámbitos diferentes de la experiencia. 


\section{Bibliografía}

Gaya, Ramón, 1992, Obra Completa, t. II, Valencia, Pre-Textos. , 2001, Naturalidad del arte (y artificialidad de la crítica), Valencia, Pre-Textos.

López-Morillas, Juan, 1956, El krausismo español, México, FCE.

López Jiménez, Luis, 1977, El naturalismo y España. Valera frente a Zola, Madrid, Alhambra.

Miralles, Enrique, 1979, La novela española de la Restauración (1875-1885): sus formas y enunciados narrativos, Barcelona, Puvill.

Oleza, Juan, 1998, "La génesis del realismo y la novela de tesis", en Romero Tobar, Leonardo (coord.), Historia de la literatura española S. XIX, t. II, Madrid, Espasa-Calpe.

Otto, Rudolf, 2001, Lo santo. Lo racional y lo irracional en la idea de Dios, trad. Fernando Vela, Madrid, Alianza.

Segovia, Tomás, 2000, Resistencia. Ensayos y notas 1997-2000, México, Ediciones Sin Nombre/UNAM.

Sennett, Richard, 2009, El artesano, trad. Marco Aurelio Galmarini, Barcelona, Anagrama.

Subirats, Eduardo, 2007, Las estrategias del espectáculo. Ensayo sobre estética y teoría crítica, Monterrey, Universidad Autónoma de Nuevo León.

Zambrano, María, 1996, Filosofía y poesía, México, FCE. 